NaIMA IfTICENE-HABANI ${ }^{1,2}$ Mahand Messaoudene ${ }^{3}$

\section{Croissance radiale et sensibilité au climat du pistachier de l'Atlas, Pistacia atlantica Desf., en Algérie}

${ }^{1}$ Université des sciences et de la technologie Houari Boumediene Faculté des sciences biologiques Laboratoire d'écologie végétale et environnement

BP 32, El-Alia

16111 Alger

Algérie

${ }^{2}$ Institut national de la recherche forestière BP 37, Chéraga

16014 Alger

Algérie

${ }^{3}$ Institut national de la recherche forestière Station régionale de Tizi Ouzou BP 30, Yakouren 15365 Tizi Ouzou Algérie

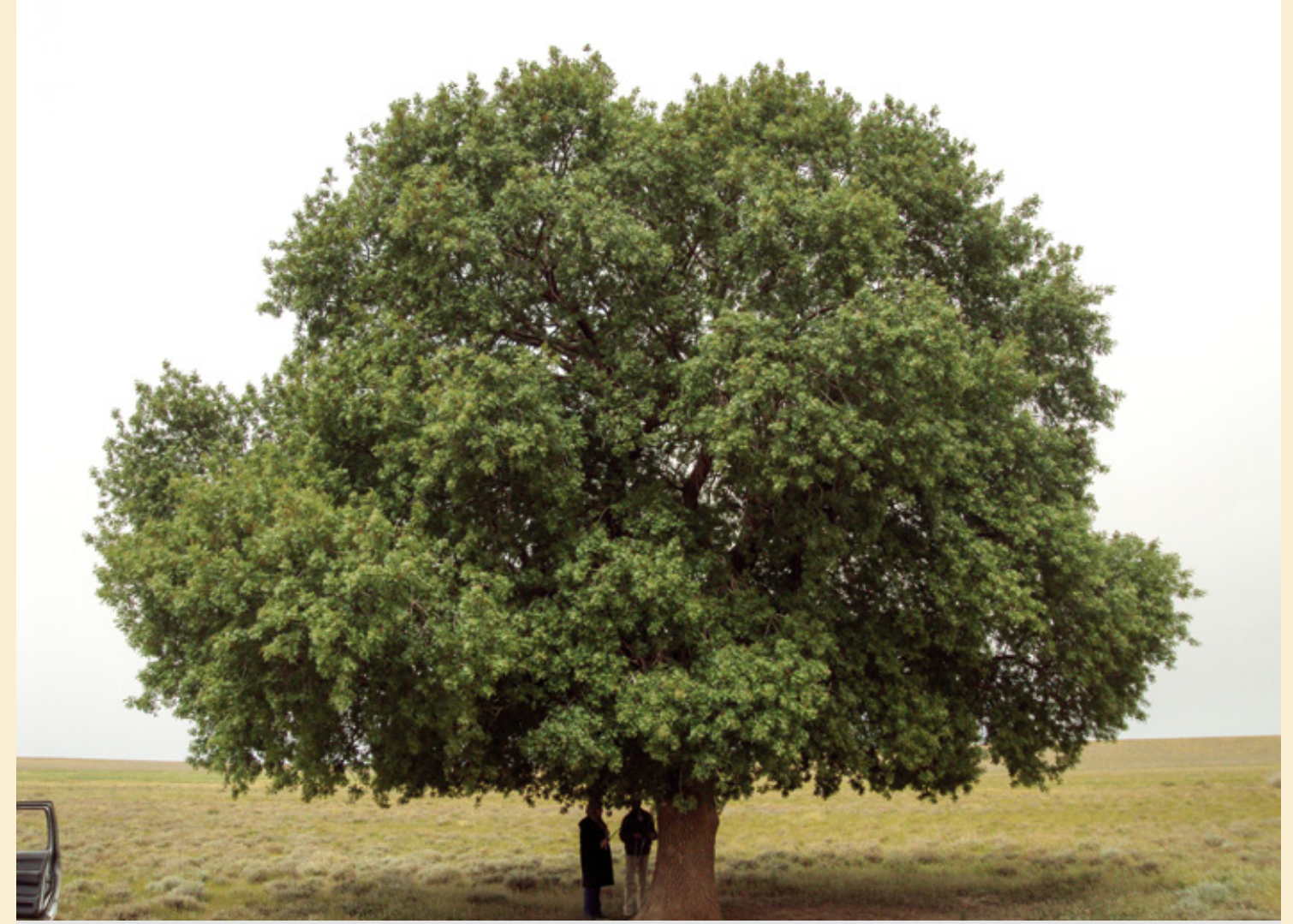

Photo 1.

Pistachier de l'Atlas, arbre majestueux en bordure d'un oued (Aïn Oussara).

Photo N. Ifticene-Habani. 


\section{RÉSUMÉ}

\section{CROISSANCE RADIALE ET SENSIBILITÉ AU CLIMAT DU PISTACHIER DE L'ATLAS, PISTACIA ATLANTICA DESF., EN ALGÉRIE}

Le pistachier de l'Atlas, Pistacia atlantica Desf., est assez peu étudié alors qu'il occupe une place importante dans les écosystèmes steppique et saharien algériens (Ahaggar: Sahara central). L'espèce constitue un cas écologique et biogéographique particulier ; son association à d'autres espèces caractéristiques des milieux saharien, aride et semi-aride indique sa très grande résistance aux changements globaux, notamment climatique. La question posée est de savoir comment réagit cet arbre aux changements climatiques actuels suivant un gradient d'aridité. Une étude dendro-éco logique a été entreprise pour identifier les facteurs climatiques régissant la croissance radiale de cette espèce. Cette approche permet de préciser à différentes échelles spatiales et temporelles la relation entre variabilité climatique et variabilité de la croissance radiale du pistachier de l'Atlas. La réponse du pistachier de l'Atlas aux évènements climatiques extrêmes a été étudiée par l'analyse des années caractéristiques. L'étude a porté sur quatre populations installées dans deux régions : la région des hautes plaines steppiques de Djelfa et la région présaharienne de Béchar. L'analyse révèle que les années caractéristiques dépendent fortement des conditions hydriques (précipitations moyennes annuelles) et thermiques (températures moyennes annuelles). Les années de forte croissance correspondent à des années humides. En revanche, les années de faible croissance correspondent à des années sèches. La réponse du pistachier de l'Atlas à la grande variabilité climatique est traduite par les valeurs élevées du coefficient de sensitivité moyenne. L'analyse des fonctions de réponse montre l'importance des précipitations dans la croissance radiale du pistachier de l'Atlas, et le rôle moindre joué par les températures dans des conditions modulées par le biotope.

Mots-clés : Pistacia atlantica, climat aride, croissance radiale, dendroécologie, pistachier de l'Atlas, climat saharien.

\section{ABSTRACT}

\section{RADIAL GROWTH AND SENSITIVITY TO CLIMATE OF THE MOUNT ATLAS MASTIC TREE, PISTACIA ATLANTICA DESF., IN ALGERIA}

Few studies have been made of the Mount Atlas mastic tree, Pistacia atlantica Desf., despite its importance as part of Algeria's steppe and desert ecosystems (Ahaggar: central Sahara). The species is of particular ecological and biogeographical interest as its association with other characteristic species of arid and semi-arid Saharan habitats point to a high degree of resistance to global changes, especially climate change. The question addressed here is to understand how this tree species is responding to climate change along an aridity gradient. A dendro-ecological study was undertaken to identify the climatic factors governing radial growth in the species. Using this approach makes it possible to identify the relationships, at different scales in space and time, between climate variability and the variability of radial growth in the Mount Atlas mastic tree. The tree's response to extreme climatic events was investigated through analyses of characteristic years. Four populations in two different regions were analysed: the high steppes around Djelfa and the pre-Saharan region around Béchar. The analysis showed that characteristic years are highly dependent on both average annual precipitation and average annual temperature. Years with high growth are the wetter years, while years with low growth are the drier years. The response of the Mount Atlas mastic tree to wide climate variability is reflected in high values for the average sensitivity coefficient. Response function analysis shows the importance of precipitation in the radial growth of the species, and the lesser importance of temperature under conditions that vary with the biotope.

Keywords: Pistacia atlantica, arid climate, radial growth, dendro-ecology, Mount Atlas mastic tree, Saharan climate.

\section{CRECIMIENTO RADIAL Y SENSIBILIDAD CLIMÁTICA DEL ALMÁCIGO, PISTACIA ATLANTICA DESF., EN ARGELIA}

El almácigo, Pistacia atlantica Desf., es muy poco estudiado a pesar del importante lugar que ocupa en los ecosistemas esteparios y saharianos argelinos (Ahaggar: Sáhara central). Esta especie es un caso ecológico y biogeográfico especial: su asociación con otras especies características de medios áridos y semiáridos saharianos muestra su gran resistencia a cambios globales, especialmente el climático. La cuestión que se plantea es saber cómo reacciona este árbol a los actuales cambios climáticos siguiendo un gradiente de aridez. Se emprendió un estudio dendrológico para identificar los factores climáticos que rigen el crecimiento radial de esta especie. Este enfoque permite detallar, a distintas escalas espaciales y temporales, la relación entre variabilidad climática y variabilidad del crecimiento radial del almácigo. Se estudió la respuesta del almácigo a fenómenos meteorológicos extremos mediante el análisis de los años característicos. El estudio se basó en cuatro poblaciones establecidas en dos regiones: la región de altas planicies esteparias de Djelfa y la región presahariana de Béchar. El análisis revela que los años característicos dependen mucho de las condiciones hídricas (precipitación promedio anual) y térmicas (temperatura promedio anual). Los años de gran crecimiento se corresponden con años lluviosos, mientras que los años de bajo crecimiento se corresponden con años secos. La respuesta del almácigo a la gran variabilidad climática se refleja en los altos valores del índice promedio de sensibilidad climática. El análisis de las funciones de respuesta muestra la importancia de las precipitaciones en el crecimiento radial del almácigo y el papel secundario que desempeñan las temperaturas en las condiciones moduladas por el biotopo.

Palabras clave: Pistacia atlantica, clima árido, crecimiento radial, dendroecología, almácigo, clima sahariano. 


\section{Introduction}

Le changement climatique est actuellement une réalité perceptible à travers la nature et l'ampleur de certains phénomènes extrêmes tels que les vagues de chaleur, les vagues de froid, les sécheresses. Le bassin méditerranéen subit, dans sa majeure partie, les effets de ce changement qui se traduisent par des événements marquants comme la sécheresse extrême des années 1990 (Hassini et al., 2015). Si, au niveau mondial, la hausse de température au XXe siècle a été de $0,74^{\circ} \mathrm{C}$, celle sur le Maghreb s'est située entre 1,5 et $2^{\circ} \mathrm{C}$ selon les régions. Quant à la baisse des précipitations, elle varie entre 10 et $20 \%$. D'autre part, de nombreuses études montrent que les projections climatiques (MCG : modèles de circulation générale) actuelles sous-estiment la hausse des températures et la baisse des précipitations sur le Maghreb. Cela montre que les pays du Maghreb vont subir, plus que d'autres, les effets du changement climatique (Tabet Aoul, 2008). En Afrique, les prévisions indiquent que l'augmentation de la température annuelle moyenne excédera $2^{\circ} \mathrm{C}$ pour atteindre 3 à $6{ }^{\circ} \mathrm{C}$ d'ici la fin du siècle. Les températures augmenteront plus rapidement dans les régions les plus arides et les précipitations diminueront en Afrique du Nord d'ici la fin du XXI ${ }^{e}$ siècle (IPCC, 2014). L'Algérie, de par sa position géographique et le caractère aride de son climat, y est particulièrement sensible (Hassini et al., 2015). Selon Tabet Aoul (2008), la principale caractéristique de ce changement consiste en une baisse des précipitations et une hausse des températures. Cette tendance, par ailleurs plus accentuée en région Ouest, favorise l'occurrence de la sécheresse qui tend, eu égard à la particularité de cette région, à devenir de plus en plus fréquente.

Les essences forestières des écosystèmes méditerranéens arides et semi-arides, mis en péril par une très forte et très ancienne pression humaine et animale, ne sont pas immuables. Le pistachier de l'Atlas (Pistacia atlantica Desf.) fait partie de ces essences forestières en danger (Amara, 2014). Cet arbre possède une amplitude écologique et une plasticité remarquables puisqu'il se rencontre depuis le cœur du Sahara jusqu'aux marges du bioclimat humide (Quézel et Médail, 2003). Sa rusticité le rend particulièrement intéressant quant à son utilisation dans les programmes de reforestation dans les zones semi-arides et arides (Belhadj, 2007). Les études sur le pistachier de l'Atlas ont surtout porté sur des aspects botaniques, biogéographiques, taxonomiques et écologiques (Emberger, 1938 ; Monjauze, 1980 ; Zohary, 1996 ; Quézel et Médail, 2003 ; Belhadj, 2007 ; Amara, 2014). Boudouaya et al. (2015) ont entrepris une étude sur la durabilité naturelle du bois du pistachier de l'Atlas. D'autres disciplines concernant la connaissance de l'autécologie de cette espèce devraient être mobilisées. En effet, les différents symptômes observés suite aux canicules sur une grande variété d'essences et dans une très large gamme de conditions stationnelles ont rappelé le rôle clé du climat dans la vitalité des écosystèmes (Bréda et al., 2004).

Notre objectif consistait à comprendre le fonctionnement de l'espèce sur la base de recherches dendroécologiques, et à connaître sa sensibilité au climat pour mieux appréhender son comportement futur et les conséquences sur sa répartition. De telles connaissances devraient en effet guider le gestionnaire dans les futurs choix des espèces à introduire dans les régions à haut risque de désertification.

\section{Matériel et méthodes}

Dans le monde, le genre Pistacia comprend onze espèces. Son aire est discontinue et compte quatre régions phytogéographiques : méditerranéenne, irano-touranienne, sino-japonaise et mexicaine (Zohary, 1996). Le pistachier de l'Atlas est essentiellement présent au Maghreb, mais aussi en Cyrénaïque, à Chypre, au Proche-Orient et aux Canaries (Quézel et Médail, 2003). La sous-espèce $P$. atlantica est endémique du grand Maghreb (Zohary, 1996).

En Algérie, le pistachier de l'Atlas apparaît du Nord jusqu'aux régions sahariennes en passant par les hauts plateaux. La limite extrême de l'espèce se trouve en plein cœur du Hoggar, où elle existe à l'état de relique (Monjauze, 1980). Les peuplements se répartissent souvent selon un mode contracté en bordure des lits d'oueds ou dans des dépressions. Il s'agit généralement de dayas où le cortège des espèces pérennes est assez pauvre, mais Ziziphus lotus est toutefois souvent bien développé en sous-strate. Les dayas sont des dépressions fermées épisodiquement, mises en eau lors de la saison froide, l'eau y stagnant de un à quatre ou cinq mois suivant les années (Quézel et Médail, 2003). Les sols profonds présentent des différences importantes en fonction de l'âge et de la situation géomorphologique de la daya (Pouget, 1977). L'espèce se régénère facilement par semis naturel, mais ces conditions sont très rarement satisfaisantes. Le plus souvent, seules les régénérations à l'abri d'épineux (Ziziphus lotus) par « effet nurserie » ou dans les anfractuosités de falaises ont quelques chances d'aboutir à des individus adultes (Quézel et Médail, 2003).

Le choix des stations a été conduit sur la base du contraste stationnel topographique et édaphique (oued et daya) des principales pistacheraies de deux régions, représentatives de chaque zone bioclimatique (semi-aride au Centre algérien et aride à l'extrême Ouest). Après investigation, il a été retenu deux stations pour chaque région : Aïn Oussara et Messaad à Djelfa, Djedida et Oum Chegag à Béchar. Aïn Oussara prend place dans le vaste domaine steppique des Hauts-Plateaux maghrébins et Messaad appartient aux régions présahariennes des dayas situées au sud de la flexure Sud-Atlasique (figure 1).

Bien que les pistacheraies de Béchar soient physionomiquement similaires à celles de Djelfa, elles se situent dans des conditions écologiques plus variées : berges et lits de cours d'eau, et cordons dunaires. Les photos 1, 2, 3 et 4 montrent le pistachier de l'Atlas dans les stations étudiées. Le tableau I récapitule les caractéristiques physiques des stations et leur situation géographique. Pour chaque région, une station météorologique représentative du climat régional a été retenue, dont les données sont complètes pour les précipitations et les températures moyennes annuelles. Les données ont été recueillies par l'Office national de météorologie (ONM) durant une période de 55 ans (1956-2011) pour Béchar et 39 ans (1972-2011) pour Djelfa. La figure 2 illustre les diagrammes ombrothermiques des deux stations météorologiques.

Les individus sélectionnés répondent aux critères établis par Stokes et Smiley (1968) : arbres plus ou moins isolés, sains et dépourvus d'anomalies graves (arbres inclinés, incendiés, blessés, étêtés...). Pour chaque arbre, trois 


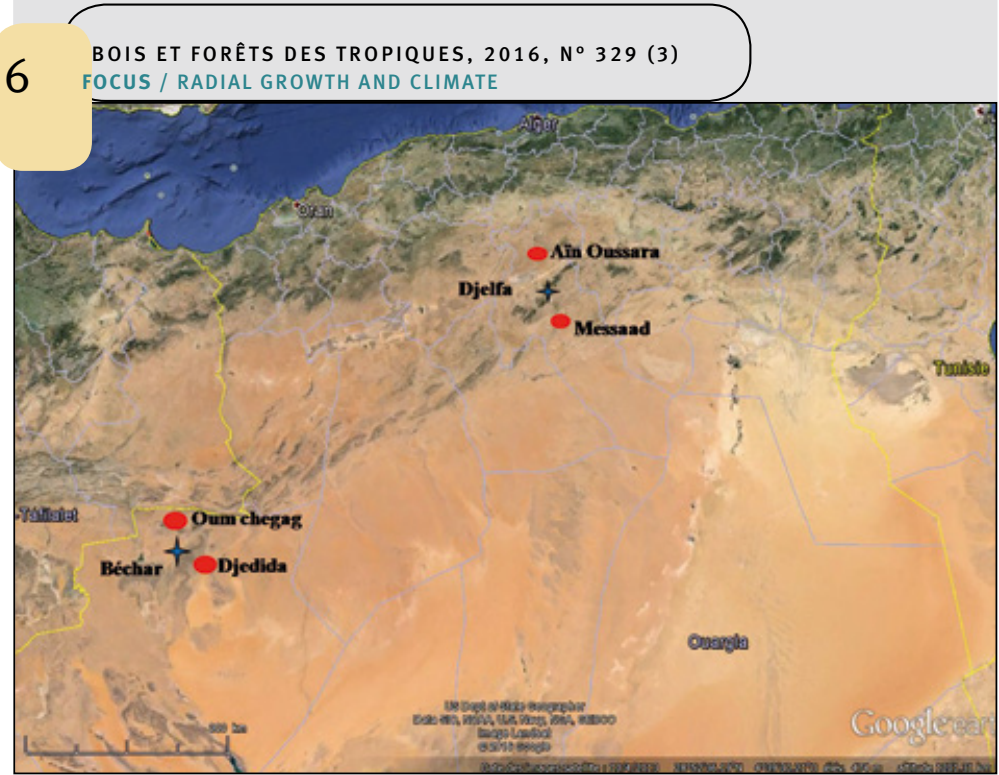

Figure 1.

Carte de situation géographique des stations d'étude

(source : Google Earth). Station échantillonnée (rond rouge).

Station météorologique (étoile bleue).

carottes ont été extraites à une hauteur de tronc égale à 1,30 m à l'aide d'une tarière de Pressler. Les prélèvements ont été effectués à $120^{\circ}$ les uns des autres, la première carotte étant orientée arbitrairement au Nord (Fritts, 1976 ; Burnel et Pélissier, 2009).

Les carottes ont été collées sur des réglettes de bois rainurées de manière à faire apparaître les cernes de croissance dans un plan transversal et éviter leur torsion, puis ont été

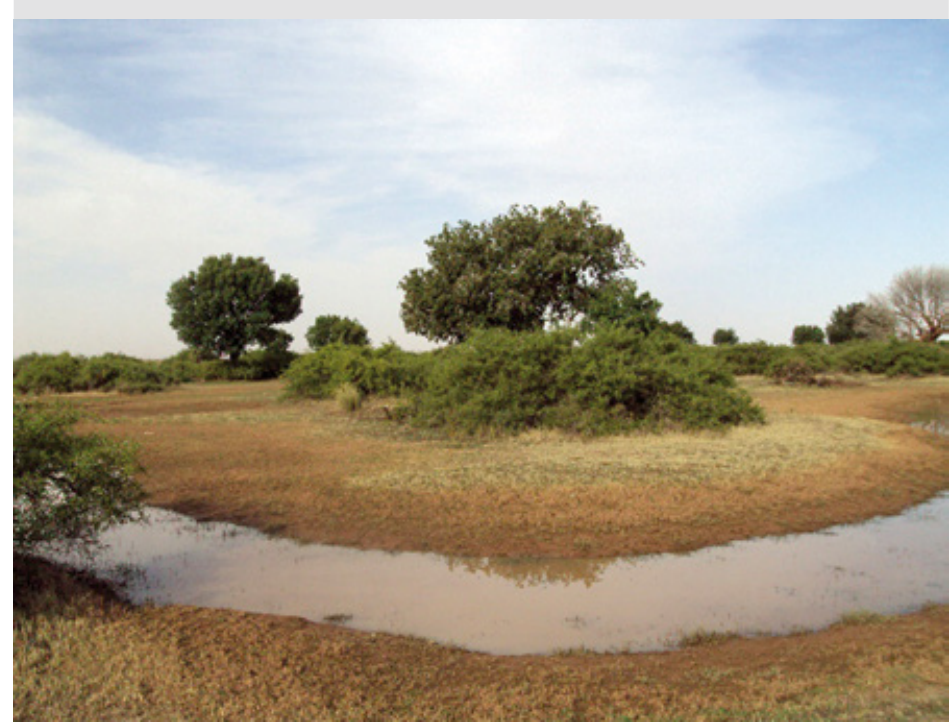

Photo 2.

Eau stagnante dans une daya (juin 2011, Messaad). Photo N. Ifticene-Habani.

poncées à l'aide d'une ponceuse à bandes de papier abrasif à grains de plus en plus fins, pour faciliter la lecture et les mesures des cernes. Les mesures des épaisseurs des cernes ont été réalisées à une précision de $1 / 1000 \mathrm{~mm}$. La largeur des cernes de chaque échantillon a été enregistrée en déplaçant, sous une loupe binoculaire, le chariot mobile (banc manuel LINTAB $^{\text {Tm5}}$ ) relié à un ordinateur équipé d'un système automatique d'enregistrement, et d'un logiciel TSAP Win ${ }^{\circledR}$

Tableau I.

Caractéristiques physiques des stations.

\begin{tabular}{|c|c|c|c|c|}
\hline Caractères & Aïn Oussara & Messaad & Djedida & Oum Chegag \\
\hline Longitude & $\begin{array}{l}2^{\circ} 53^{\prime} 508^{\prime \prime} \\
\text { et } 3^{\circ} 18^{\prime} 067^{\prime \prime}\end{array}$ & $\begin{array}{l}3^{\circ} 37^{\prime} 170^{\prime \prime} \\
\text { et } 3^{\circ} 47^{\prime} 682^{\prime \prime}\end{array}$ & $\begin{array}{l}1^{\circ} 44^{\prime} 935^{\prime \prime} \\
\text { et } 1^{\circ} 54^{\prime} 977^{\prime \prime}\end{array}$ & $\begin{array}{l}2^{\circ} 17^{\prime} 999^{\prime \prime} \\
\text { et } 2^{\circ} 19^{\prime} 365^{\prime \prime}\end{array}$ \\
\hline Latitude & $\begin{array}{l}35^{\circ} 30^{\prime} 609^{\prime \prime} \\
\text { et } 35^{\circ} 12^{\prime} 244^{\prime \prime}\end{array}$ & $\begin{array}{l}33^{\circ} 55^{\prime} 364^{\prime \prime} \\
\text { et } 34^{\circ} 00^{\prime} 814^{\prime \prime}\end{array}$ & $\begin{array}{l}31^{\circ} 44^{\prime} 182^{\prime \prime} \\
\text { et } 31^{\circ} 59^{\prime} 423^{\prime \prime}\end{array}$ & $\begin{array}{l}32^{\circ} 04^{\prime} 593^{\prime \prime} \\
\text { et } 32^{\circ} 06^{\prime} 006^{\prime \prime}\end{array}$ \\
\hline Altitude (m) & 700 à 900 & 600 à 700 & 700 à 1000 & 950 à 990 \\
\hline Pente (\%) & 2 à 5 & 0 à 2 & 5 à 10 & 2 à 5 \\
\hline Élément floristique & $\begin{array}{l}\text { Ziziphus lotus } \\
\text { Artemisia herba-alba } \\
\text { Stipa tenacissima }\end{array}$ & $\begin{array}{l}\text { Ziziphus lotus } \\
\text { Artemisia herba-alba } \\
\text { Stipa tenacissima }\end{array}$ & $\begin{array}{l}\text { Launaea arborescens } \\
\text { Retama retam } \\
\text { Acacia raddiana }\end{array}$ & $\begin{array}{l}\text { Ziziphus lotus } \\
\text { Artemisia herba-alba } \\
\text { Retama retam }\end{array}$ \\
\hline Élément topographique & Oueds & Dayas & Oueds & Dayas \\
\hline Élément géologique & $\begin{array}{l}\text { Alluvions récentes } \\
\text { du quaternaire }\end{array}$ & $\begin{array}{l}\text { Crétacé inférieur } \\
\text { continental }\end{array}$ & Quaternaire récent & $\begin{array}{l}\text { Dépôts de dunes } \\
\text { sur quaternaire }\end{array}$ \\
\hline Bioclimat & Aride frais & Aride frais & Saharien tempéré & Saharien tempéré \\
\hline Texture du sol & Sablonneuse & Limono-argileuse & Sablo-limoneuse & Argilo-limono-sableuse \\
\hline $\begin{array}{l}\text { Hauteur totale moyenne } \\
\text { du peuplement }(\mathrm{m})\end{array}$ & 10,92 & 11,01 & 8,77 & 7,53 \\
\hline $\begin{array}{l}\text { Circonférence moyenne } \\
\text { du peuplement }(\mathrm{m})\end{array}$ & 2,7 & 2,59 & 1,91 & 1,12 \\
\hline Nombre de cernes mesurés & 46 & 46 & 59 & 65 \\
\hline Écart-type & 7 & 6 & 10 & 14 \\
\hline
\end{tabular}




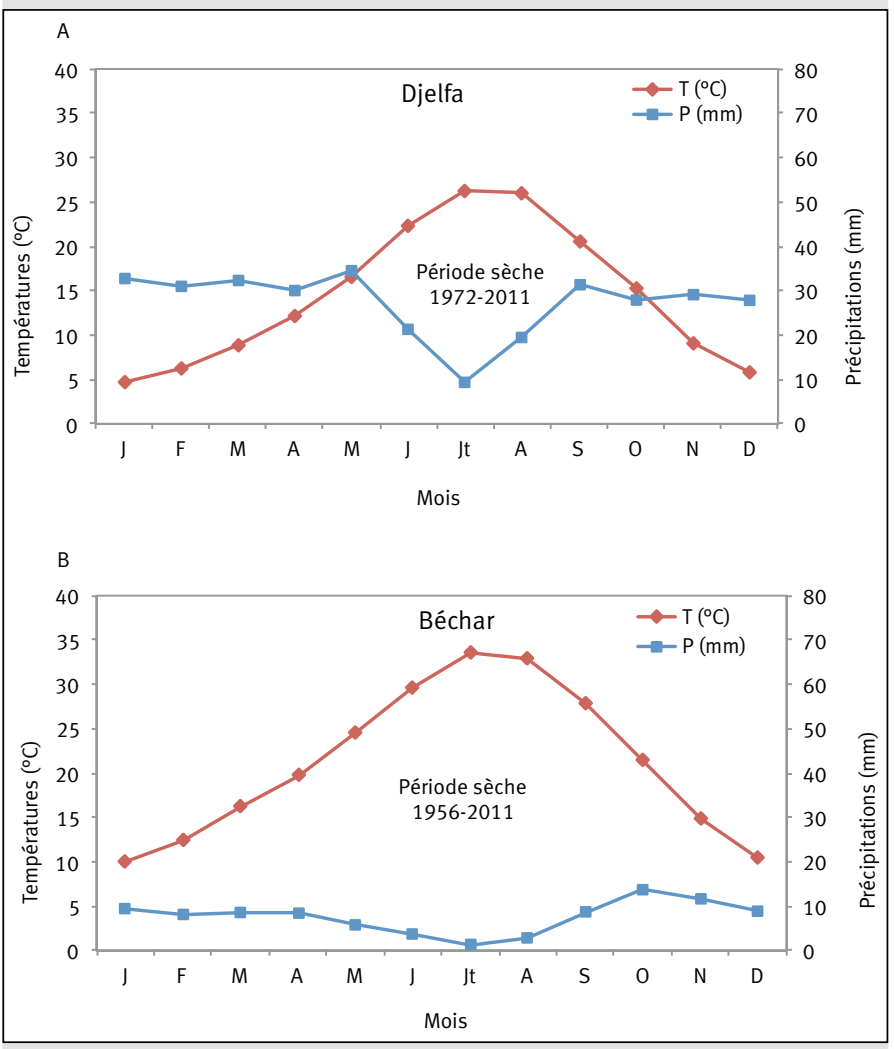

Figure 2.

Diagrammes ombrothermiques de Bagnouls et Gaussen des stations météorologiques.

(Time Series Analysis and Presentation). Les mesures ont été effectuées au laboratoire de la qualité du bois à la station régionale de l'Institut national de la recherche forestière d'Azzazga (Tizi Ouzou). L'interdatation, étape fondamentale qui consiste à attribuer à chaque cerne l'année exacte de sa formation, s'est avérée très difficile. Les difficultés étaient liées à la présence dans certains échantillons de zones à cernes très minces voire absents, ou à l'anatomie du bois à pores diffus, ce qui a rendu la limite d'un cerne à l'autre difficile à identifier. Sur les 81 arbres échantillonnés à Djelfa (50 pour Aïn Oussara et 31 pour Messaad), seuls 36 ont été interdatés (44\%); 21 arbres sur 77 (47 à Djedida et 30 à Oum Chegag) l'ont été à Béchar ( $28 \%$ ). Une autre anomalie a été observée dans le bois du pistachier : s'agissant de la présence de faux cernes ou cernes doubles dans le bois initial et parfois dans le bois final (photos 5, 6 et 7). Ce phénomène a été détecté en région méditerranéenne sur différentes espèces. Safar (1994) note la présence de cernes doubles chez Pinus halepensis dans la région de Djelfa. Cherubini et al. (2003) identifient des cernes doubles sur Arbutus unedo et Quercus ilex dans le Lajatico (Toscane, Italie) et attribuent ces derniers au caractère xérique de la station.

Divers paramètres statistiques ont également été calculés afin d'évaluer la qualité et la force du signal climatique contenu dans les séries de cernes. La sensitivité moyenne (SM) et le coefficient d'autocorrélation (AC) d'ordre 1 ont été calculés pour évaluer la variabilité moyenne d'épaisseur entre deux cernes successifs et exprimer l'importance des

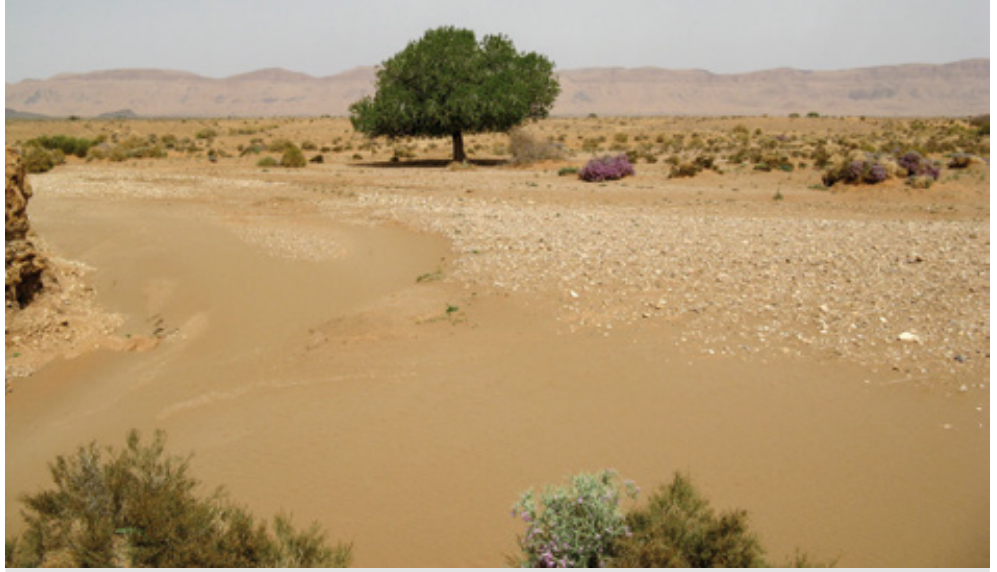

Photo 3.

Pistachier de l'Atlas, en bordure d'un oued à Djedida.

Photo N. Ifticene-Habani.

changements climatiques à court terme affectant ces derniers (Fritts, 1976). L'EPS (expressed signal population) a été calculé pour estimer la force du signal contenu dans les cernes étudiés (Wigley et al., 1984).

L'analyse de la réponse aux événements climatiques extrêmes (fortes sécheresses estivales, froids hivernaux intenses) par le calcul des années caractéristiques, aussi appelées pointer years (Fritts, 1976 ; Schweingruber et al., 1990), a été réalisée à l'aide de l'application POINTER développée sous le logiciel libre R ( $R$ Development Core Team, 2011) par Mérian (2012a). POINTER permet de calculer les années caractéristiques à partir des épaisseurs brutes de cernes interdatées, selon la méthode développée par Becker (1989) :

$E R_{t}=\frac{L C_{t}-L C_{t-1}}{L C_{t-1}}$

ER étant l'écart relatif de la largeur de cerne LC entre les années $t$ et $t-1$.

Les années caractéristiques correspondent aux dates pour lesquelles au moins $75 \%$ des arbres du peuplement considéré présentent une variation relative de croissance d'au moins $10 \%$ par rapport à l'année précédente. L'année est dite positive en cas de croissance plus forte et négative dans le cas contraire. Le pourcentage de variation est appelé écart relatif moyen (ERM). Nous considérons comme années exceptionnelles sèches ou fraîches celles pour lesquelles le total des précipitations ou des températures moyennes est inférieur à la moyenne de la série climatique, diminuée de la valeur de l'écart-type (Safar, 1994). De manière similaire, nous considérons comme années exceptionnelles humides ou chaudes celles pour lesquelles le total des précipitations ou des températures est supérieur à la moyenne de la série climatique, augmentée de la valeur de l'écart-type (Safar, 1994). 


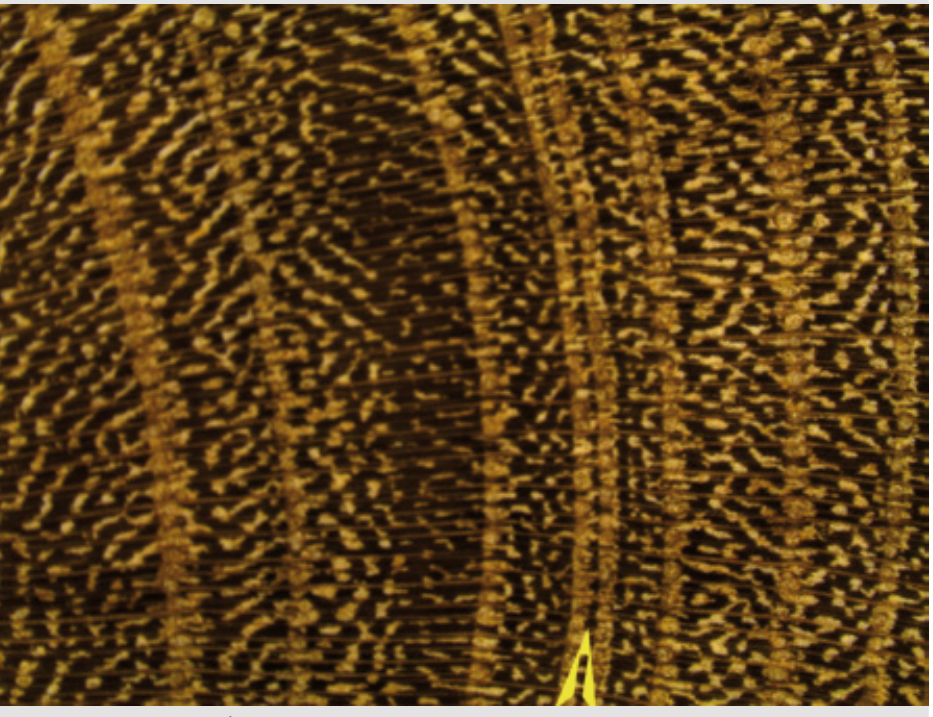

Photo 6.

Faux cernes (flèche jaune) provoqués par la sécheresse au cours de l'activité cambiale (grossissement : 0,63 x 10). Photo N. Ifticene-Habani.

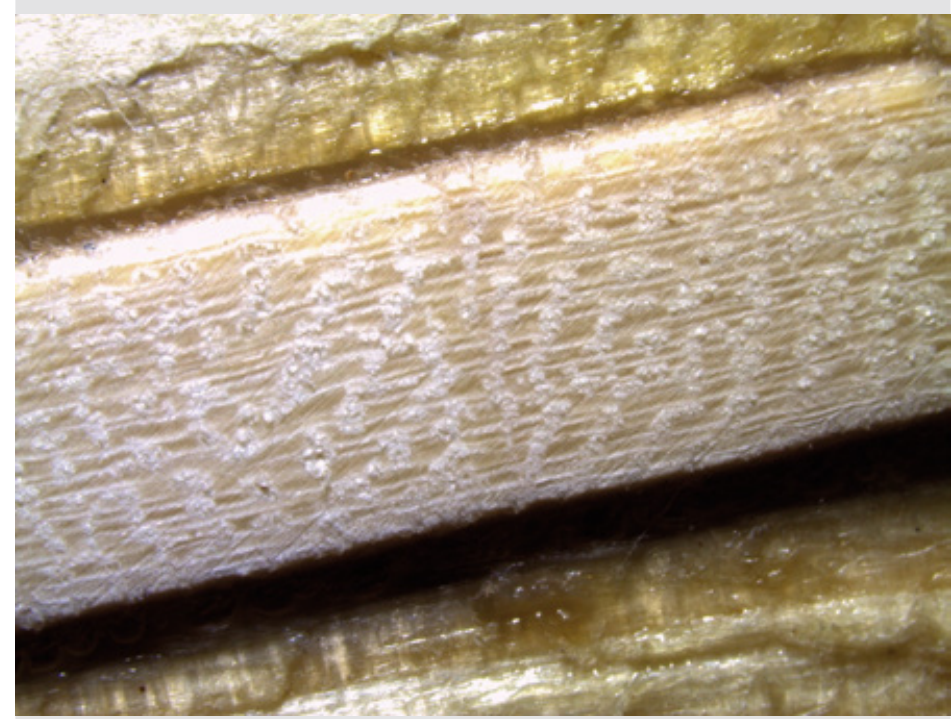

Photo 5.

Carotte très difficile à interdater : bois à pores diffus (grossissement : 0,63 $\times 10$ ). Photo N. Ifticene-Habani.

Les fonctions de réponse (Fritts, 1976) ont été calculées afin d'identifier les facteurs climatiques significativement corrélés à l'épaisseur des cernes (Guiot, 1991). L'application DENDRO développée sous le logiciel libre $R$ par Mérian (2012a) a été utilisée pour établir la relation entre l'épaisseur des cernes des pistachiers et les facteurs climatiques. DENDRO permet de standardiser les séries brutes d'épaisseurs de cerne selon le processus classique de la double pondération afin d'éliminer les signaux non liés au climat (Cook, 1985 ; Mérian, 2012a), mais aussi de calculer des fonctions de réponse et de corrélation entre les indices de croissance et les régresseurs climatiques (Cook et Kairiukstis, 1990 ; Fritts, 1976 ; Guiot, 1991). Ces fonctions reposent sur une approche de type Bootstrap permettant de tester simultanément les coefficients de régression ou

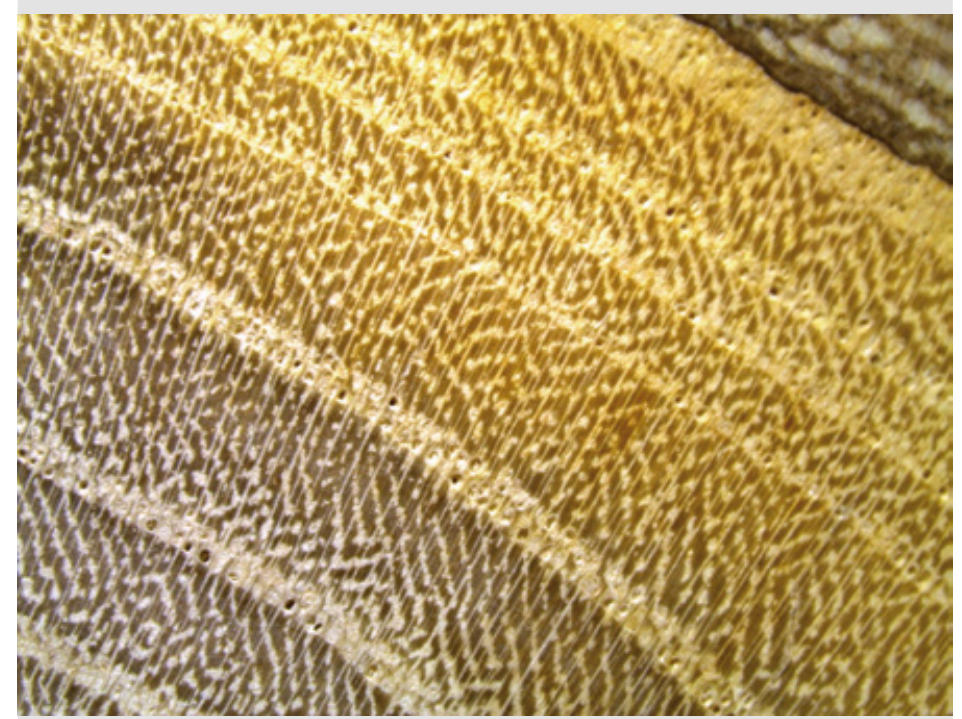

\section{Photo 7.}

Échantillon à limites de cernes très lisibles (grossissement : 0,63 x 10).

Photo N. Ifticene-Habani.

de corrélation (BCC) et la stabilité des fonctions entre des échantillons indépendants (tirage aléatoire de $x$ années avec remise) (Guiot, 1991). Les variables climatiques utilisées couvrent la période allant de septembre de l'année précédente $t-1$ à août de l'année en cours $t$ (12 mois) pour les températures moyennes mensuelles et les précipitations moyennes mensuelles. Ainsi, chaque chronologie moyenne CRN est confrontée à un jeu de données climatiques constitué de 24 régresseurs. La mise en forme et la standardisation des séries climatiques ont été réalisées par l'application CLIMAT (Mérian, 2012a) pour leur utilisation dans DENDRO. La finalité de la standardisation des séries climatiques est d'obtenir des séries d'indices stationnaires dans lesquelles seul le signal haute fréquence est conservé (Fritts, 1976 ; Schweingruber, 1996). 


\section{Résultats et discussion}

\section{Variation de l'épaisseur brute des cernes}

Les valeurs de l'épaisseur des cernes (EMC) ont été reportées dans le tableau II. Les valeurs élevées des écartstypes et des coefficients de variation indiquent une grande fluctuation de la croissance radiale entre individus de la même station, et traduisent aussi la variabilité temporelle à l'intérieur d'une même chronologie. La largeur du cerne des pistacheraies de Djelfa est sensiblement supérieure à celle de Béchar. Ainsi, les particularités stationnelles induisent des variations différentielles au niveau de l'intensité de la réaction des arbres aux facteurs de l'environnement qui fluctuent d'une année à l'autre. Cette réaction différenciée peut être attribuée soit à un climat moins aride à Djelfa qu'à Béchar, soit à la nature particulière du biotope (Safar, 1994). Les populations de Aïn Oussara et de Djedida, implantées sur les bordures des lits d'oueds, ne bénéficient pas de conditions susceptibles d'emmagasiner l'eau des pluies pour une longue durée et par conséquent de tamponner les effets du facteur climatique limitant. Dans notre cas, le facteur limitant la croissance radiale du pistachier peut être attribué à l'eau (précipitations). Par contre, les populations de Messaad et d'Oum Chegag, situées au niveau des dayas, bénéficient de conditions favorables au stockage de l'eau.

La sensitivité moyenne (sens1) calculée sur les chronologies de synthèses brutes varie d'une région à l'autre. Djelfa apparaît plus sensible (sens1 $=0,49$ à Messaad et 0,42 à Aïn Oussara). À Béchar, les sensitivités sont très proches dans les deux stations (sens $1=0,35$ à Djedida et 0,36 à Oum Chegag). Ces valeurs sont supérieures au seuil moyen de 0,20 pour les espèces méditerranéennes, ce qui démontre une bonne réactivité du pistachier aux variations interannuelles de l'environnement. Les coefficients de sensitivité moyenne les plus élevés sont toujours observés dans les régions semi-arides. Safar (1994) obtient pour le pin d'Alep de Djelfa des valeurs de 0,14 à 0,31 et attribue le caractère sensitif de ces régions aux influences désertiques. Le coefficient d'autocorrélation d'ordre 1 est plus faible à Djelfa qu'à Béchar mais n'excède pas 0,5 . Ce coefficient exprime le phénomène de "persistance » dans la mesure où le cerne de l'année $t$ se trouve déjà prédéterminé en grande partie par la croissance et les conditions climatiques de l'année antérieure $t-1$ qui déterminent le niveau des réserves (carbohydrates et réserves hydriques, en particulier) disponibles au moment du démarrage de la croissance (Fritts, 1976). À Béchar, le coefficient d'autocorrélation plus élevé $(0,5)$ attribue au jeu des facteurs internes un rôle plus important qu'à Djelfa.

\section{Années caractéristiques et climat}

Le tableau III révèle un nombre plus élevé d'années caractéristiques au niveau des dayas qu'au niveau des lits d’oueds. Dans la majorité des cas, les années caractéristiques correspondent à des conditions particulièrement sèches ou pluvieuses, à l'exception de 1972 et 2001 pour la région steppique de Djelfa et 1967 pour la région présaharienne de Béchar. Le froid enregistré en 1972, 1974, 1975 et 1992 à Djelfa a entraîné une faible croissance des pistachiers. En revanche, en 1976, malgré les basses températures hivernales, les pistachiers ont produit un cerne épais en raison des précipitations supérieures à la moyenne $(182,14 \mathrm{~mm})$. Pour les deux stations, le déficit hydrique et la chaleur de l'année 2000 enregistrés dans les hautes plaines steppiques ont causé un déficit de croissance de $53 \%$ et $56 \%$. Une reprise spectaculaire est observée en 2001 suite à la diminution du déficit hydrique (de - 175,36 mm à $-89,96 \mathrm{~mm})$. À Béchar, le pistachier a produit un cerne épais en 1965, 1994, 2006 et 2008 à Oum Chegag, où la pluviosité a été supérieure à la moyenne de 148,24 mm, $67,34 \mathrm{~mm}, 73,94 \mathrm{~mm}$ et $145,4 \mathrm{~mm}$. À l'inverse, le pistachier a produit un cerne mince en 2000, 2001 et 2010, années à déficits hydriques respectifs de $-35,66 \mathrm{~mm},-76,56 \mathrm{~mm}$ et $-48,96 \mathrm{~mm}$. L'année 2000, marquée par des conditions chaudes et sèches, est une année caractéristique négative dans les quatre stations.

Afin de mieux comprendre les conditions climatiques qui ont prévalu lors des années caractéristiques, nous avons moyenné les précipitations et températures moyennes des années caractéristiques positives et négatives par rapport à la moyenne de chaque région. Pour Djelfa, les années caractéristiques négatives se différencient par des précipitations largement supérieures à la moyenne en mars et avril. Ainsi en 1972, $37 \%$ des pluies de l'année ont été enregistrées au printemps, $60 \%$ en 1992 et $43 \%$ en 2004 . Les années caractéristiques positives semblent quant à elles offrir des 
Tableau III.

Années caractéristiques et années exceptionnelles. Les années caractéristiques correspondent aux dates pour lesquelles au moins $75 \%$ des arbres du peuplement présentent une variation relative de croissance d'au moins $10 \%$ par rapport à l'année précédente. Une année exceptionnelle (sèche ou humide, chaude ou fraîche) est une année qui a reçu des précipitations ou des températures dont le total annuel est inférieur ou supérieur d'un écart à la moyenne.

Année

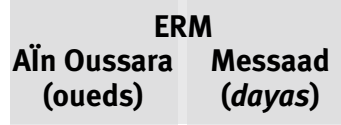

1956

1957

1958

1960

1961

1965

1966

1967

1970

1972

1973

1974

1975

1976

1977

1980

1981

1984

1985

1986

1987

1988

1990

1991

1992

1994

1995

1997

2000

2001

2002

2003

2004

2005

2006

2007

2008

2010

Nombre AC+

Nombre AC-

\begin{tabular}{|c|c|}
\hline & \\
\hline & \\
\hline & \\
\hline & \\
\hline & \\
\hline & \\
\hline-67 & \\
\hline & \\
\hline & -29 \\
\hline & -29 \\
\hline & 199 \\
\hline 58 & -67 \\
\hline & 40 \\
\hline
\end{tabular}

\section{Année exceptionnelle $\mathrm{T}\left({ }^{\circ} \mathrm{C}\right) \quad \mathrm{P}(\mathrm{mm})$}

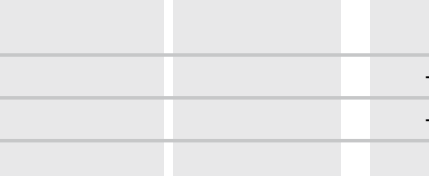

Djedida Oum Chegag (oueds) (daya)
Année exceptionnelle

$\mathrm{T}\left({ }^{\circ} \mathrm{C}\right) \quad \mathrm{P}(\mathrm{mm})$

\begin{tabular}{r|c|c|c|} 
& 26 & $-\mathbf{0 , 6 6}$ & $-\mathbf{3 5 , 0 6}$ \\
-12 & 27 & $-0,66$ & 13,24 \\
-20 & & &
\end{tabular}

$\begin{array}{lll}-23 & 0,34 & -35,06\end{array}$

$\begin{array}{llll}-27 & -33 & 0,32 & -50,26\end{array}$

$\begin{array}{lll}159 & -0,27 & 148,24\end{array}$

$-33 \quad-19,86$

$-29$

$-36$

$-27$

$-1,02 \quad 17,24$

34

$-1,57 \quad 151,44$

$-20$

$-0,20 \quad-24,26$

$-0,91$

$-1,16$

$-2,28$

$-35$

$-0,54$

$-19,16$

182,14

$-55,86$

$-55,86$

\begin{tabular}{|c|c|c|c|c|c|c|c|}
\hline & & & & & -35 & 0,81 & $-74,86$ \\
\hline & & & & & -20 & $-0,73$ & $-69,76$ \\
\hline \multirow[t]{3}{*}{-33} & -42 & & & & & & \\
\hline & & & & -22 & & $-0,46$ & \\
\hline & 144 & & & & & & \\
\hline \multicolumn{8}{|l|}{58} \\
\hline & & & & & 39 & 0,50 & $-18,06$ \\
\hline & 60 & $-0,83$ & 123,64 & & & & \\
\hline & -44 & $-1,08$ & & & -46 & $-1,35$ & $-53,96$ \\
\hline & 111 & & & & 113 & 0,07 & 67,34 \\
\hline & -25 & & $-73,66$ & & & & \\
\hline \multicolumn{8}{|l|}{118} \\
\hline-53 & -56 & 0,66 & $-175,36$ & -26 & -39 & 0,32 & $-35,66$ \\
\hline 158 & 171 & 1,16 & $-89,96$ & & -41 & 1,62 & $-76,56$ \\
\hline & -31 & & $-113,36$ & & & & \\
\hline & 64 & & & & 37 & & \\
\hline & -35 & & & & & & \\
\hline & 71 & & & & & & \\
\hline & & & & & 33 & 0,65 & 73,94 \\
\hline & & & & 29 & 52 & & \\
\hline & & & & & 37 & 0,17 & 145,4 \\
\hline & & & & & -26 & 1,72 & $-48,96$ \\
\hline
\end{tabular}

\begin{tabular}{l|l|l}
9 & $(+)$ Chaude & $(+)$ Humide \\
8 & $(-)$ Fraîche & $(-)$ Sèche
\end{tabular}

1

10

(+) Chaude

(+) Humide

6

13

(-) Fraîche

(-) Sèche

$\mathrm{AC}-$ : années caractéristiques négatives ; $\mathrm{AC}+$ : années caractéristiques positives ; italique : action directe ; non italique : action inverse ; $\mathrm{T}$ : température moyenne annuelle (exprimée en ${ }^{\circ} \mathrm{C}$ ) ; $\mathrm{P}$ : précipitations moyennes annuelles (exprimées en $\mathrm{mm}$ ). ; Les données en gras indiquent les années où les populations ont réagi aux conditions particulièrement sèches ou pluvieuses ; Les années qui ne sont figurées ni en italiques ni en caractères gras correspondent aux années où les populations ont réagi soit positivement ou négativement sans que ces dernières ne soient des années caractéristiques positives ou négatives.

Pour l'année 1957, les populations de Béchar ont réagi de manière différente : difficile donc de spécifier cette année. 


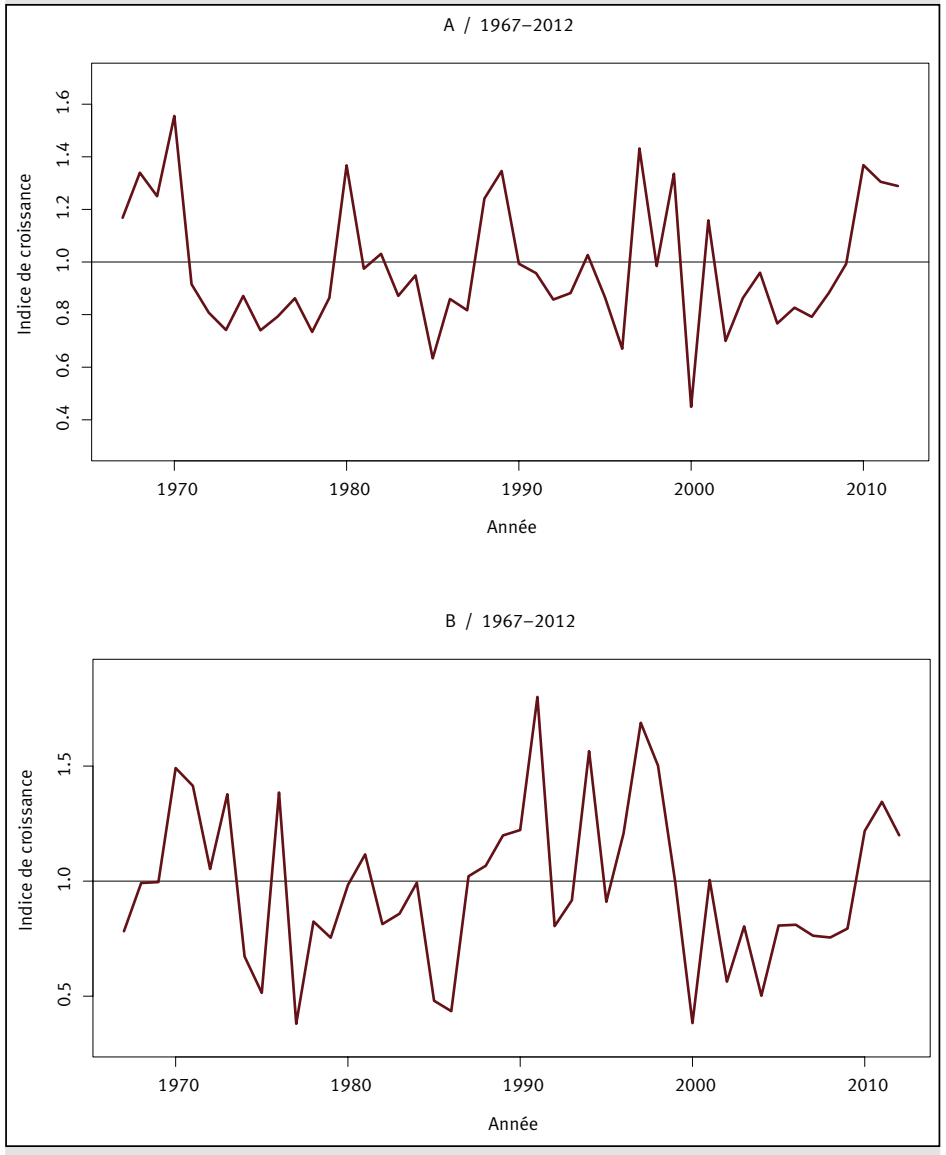

Figure 3.

Le graphique montre les indices de croissance des chronologies moyennes issues de la standardisation ; ces indices sont obtenus par une double standardisation de la largeur de cerne brute (Cook, 1985), pour la période de 1967 à 2012.

précipitations assez élevées en automne et parfois en hiver (55\% de pluies automnales en 1994, $50 \%$ en 2001 et $53 \%$ en 2005). À Béchar, la distribution des pluies en fonction des saisons ne semble pas avoir un effet sur les années caractéristiques excepté en 2010 où 40,5\% des pluies ont été enregistrées en été. Les années caractéristiques négatives sont également caractérisées par des températures inférieures à la moyenne en été. Pour Djelfa, $-2,71{ }^{\circ} \mathrm{C}$ par rapport à la moyenne était enregistré au printemps en 1972 $\left(-4,03{ }^{\circ} \mathrm{C}\right.$ en mai) et $-2,1^{\circ} \mathrm{C}$ en été $\left(-2,27^{\circ} \mathrm{C}\right.$ en août). Le froid intense de l'été $1977\left(-4,1^{\circ} \mathrm{C}\right)$ a réduit la croissance à Messaad de $67 \%$. À Béchar, la majorité des années caractéristiques négatives sont caractérisées par des températures inférieures à la moyenne en août (de $-0,15^{\circ} \mathrm{C}$ à $\left.-1,65^{\circ} \mathrm{C}\right)$ à l'exception de $2001\left(+1,15^{\circ} \mathrm{C}\right.$ en mars $)$ et $2010\left(+1{ }^{\circ} \mathrm{C}\right.$ en mai). À l'inverse, le pistachier a répondu positivement aux années à été chaud, à l'exemple de $1980\left(+10,43^{\circ} \mathrm{C}\right.$ en août), 1987 ( $+1,41^{\circ} \mathrm{C}$ en août) et 1994 ( $+2^{\circ} \mathrm{C}$ en août), au cours desquelles la croissance a été supérieure respectivement de 40 à $58 \%, 144 \%$ et $118 \%$. Selon Mérian (2012b), la variation des ERM entre les populations est certainement liée à la fois à une variation effective de la réponse à un même stress d'une population à l'autre et à une variation de l'intensité de ce stress.

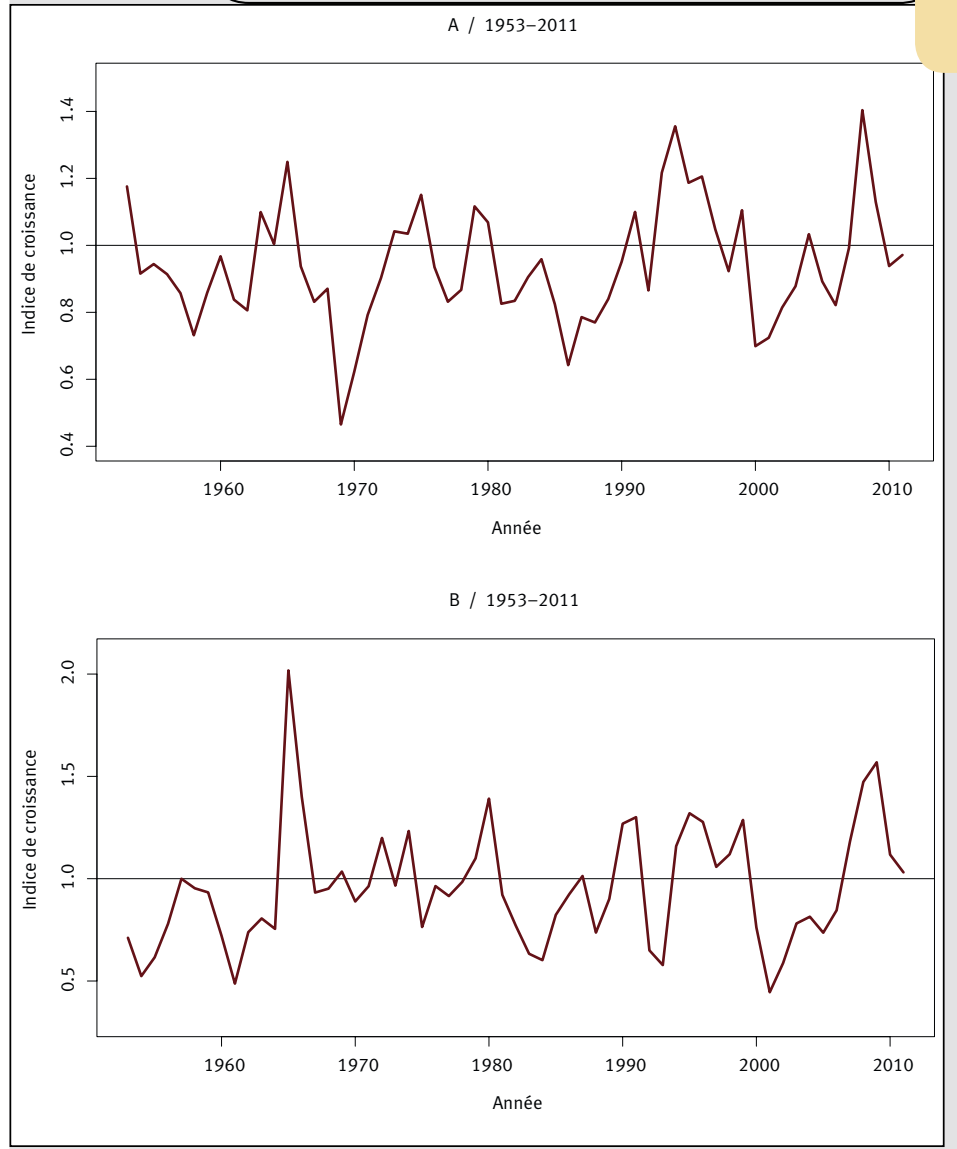

Figure 4.

Le graphique montre les indices de croissance des chronologies moyennes issues de la standardisation ; ces indices sont obtenus par une double standardisation de la largeur de cerne brute (Cook, 1985), pour la période de 1953 à 2011.

\section{Réponse au climat}

L'élimination de la tendance de la croissance liée à l'âge, à l'effet géométrique lié à l'augmentation de diamètre du tronc et à d'autres variables fluctuant au pas de temps annuel (insectes défoliateurs, rythme des fructifications et incendies) par la standardisation a permis de mettre en évidence les années exceptionnelles de la croissance. Ces années correspondent à un indice de croissance minimal inférieur à 1 pour les cernes minces et supérieur à 1 pour les cernes épais par rapport à la valeur moyenne de 1 .

Les figures 3 et 4 mettent en évidence le caractère plus au moins synchrone des variations interannuelles de l'indice de croissance pour les deux populations au sein de la même région. On constate un maximum de croissance en 1970 à Aïn Oussara, 1991 à Messaad, 2008 à Djedida et 1965 à Oum Chegag. Une crise de croissance en 2000 pour les deux populations de Djelfa, en 1969 à Djedida et en 2001 à Oum Chegag. Ces résultats confirment l'influence des variables climatiques dans la détermination de la croissance radiale du pistachier.

Les sensitivités moyennes calculées sur les chronologies standardisées sont plus faibles que celles calculées sur les séries brutes (tableau IV). En effet, sur les données brutes, les variations de croissance intègrent l'ensemble 


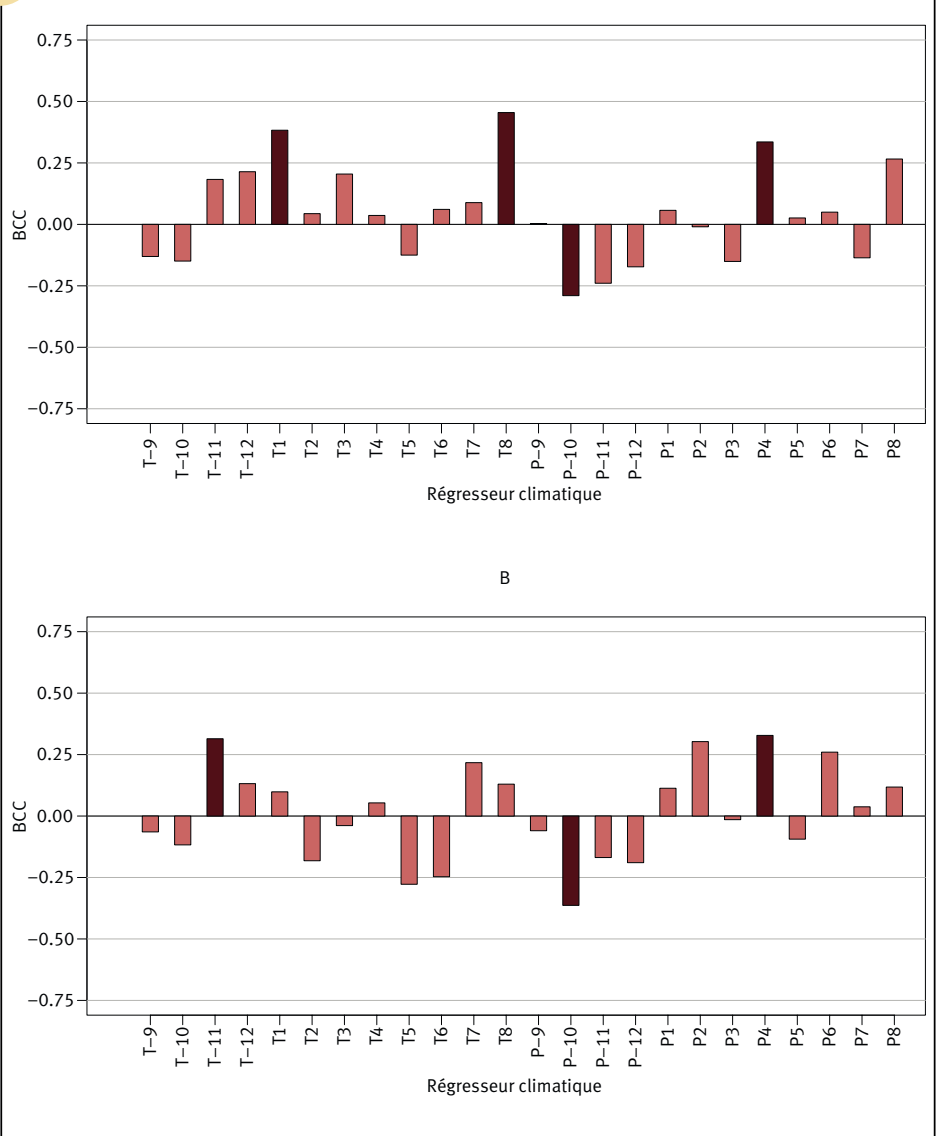

Figure 5.

BCC : coefficient de corrélation de type Bootstrap.

$\mathrm{T}$ : température ; $\mathrm{P}$ : précipitations ; les mois sont indiqués par des chiffres (1 pour janvier, etc.), les valeurs négatives correspondant aux mois de l'année précédente. Les bâtons foncés indiquent un coefficient significatif au seuil choisi par l'utilisateur (ici, $95 \%$ ).
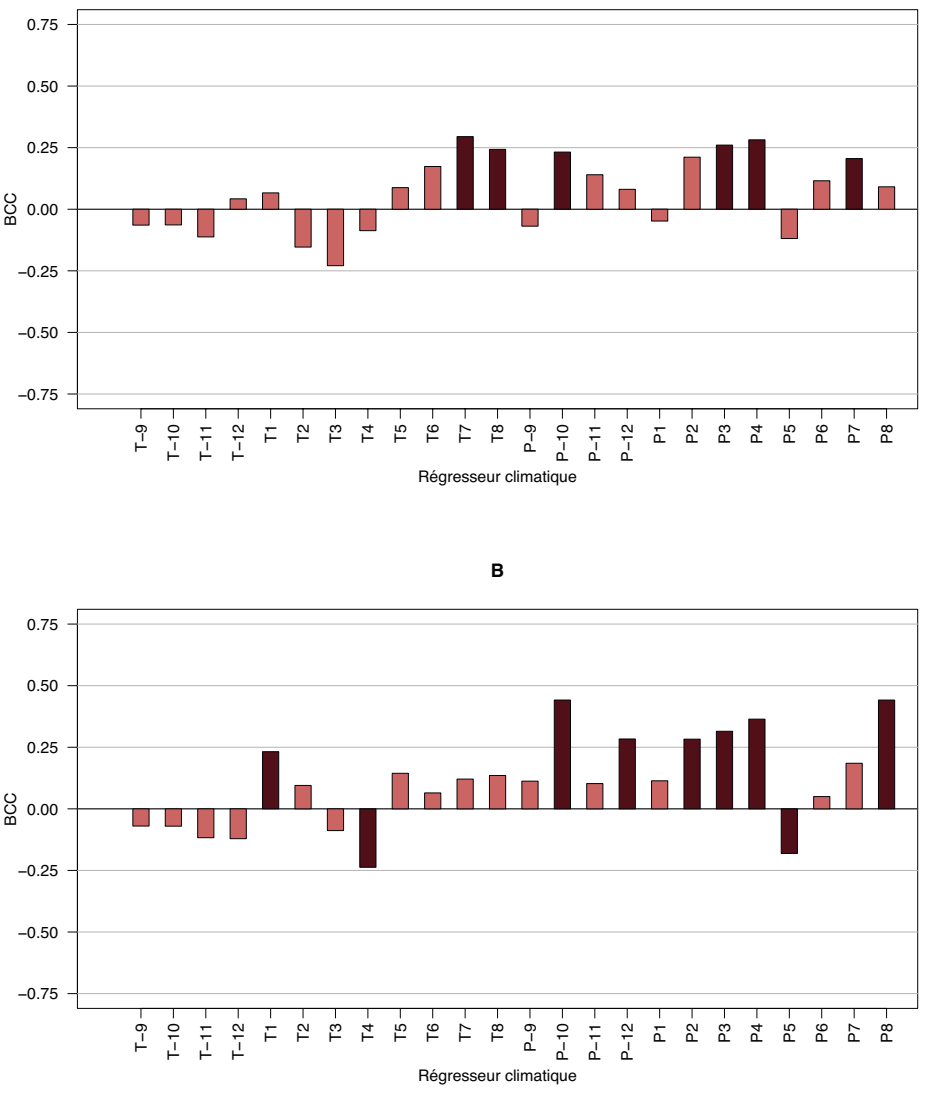

Figure 6.

BCC : coefficient de corrélation de type Bootstrap.

$\mathrm{T}$ : température ; $\mathrm{P}$ : précipitations ; les mois sont indiqués par des chiffres (1 pour janvier, etc.), les valeurs négatives correspondant aux mois de l'année précédente. Les bâtons foncés indiquent un coefficient significatif au seuil choisi par l'utilisateur (ici, $95 \%$ ).
Tableau IV.

Paramètres dendrochronologiques des chronologies standardisées.

\begin{tabular}{|c|c|c|c|c|}
\hline Paramètres & $\begin{array}{l}\text { Aiin Oussara } \\
\text { (oueds) }\end{array}$ & $\begin{array}{l}\text { Messaad } \\
\text { (dayas) }\end{array}$ & $\begin{array}{l}\text { Djedida } \\
\text { (oueds) }\end{array}$ & $\begin{array}{l}\text { Oum Chegag } \\
\text { (daya) }\end{array}$ \\
\hline IC moyen & 0,98 & 0,98 & 0,94 & 0,96 \\
\hline IC min. & 0,45 & 0,38 & 0,47 & 0,45 \\
\hline IC max. & 1,56 & 1,80 & 1,40 & 2,02 \\
\hline ET & 0,25 & 0,35 & 0,18 & 0,29 \\
\hline sens1 & 0,23 & 0,35 & 0,15 & 0,23 \\
\hline ar1 & 0,19 & 0,25 & 0,49 & 0,45 \\
\hline eps & 0,75 & 0,90 & 0,49 & 0,80 \\
\hline
\end{tabular}

des signaux liés non seulement aux facteurs du milieu mais également aux facteurs endogènes (âge, facteurs stationnels, etc.). Par contre, les sensitivités calculées sur les séries standardisées reflètent les effets propres aux facteurs du milieu (climat, notamment). Les sensitivités varient de 0,23 à 0,35 à Djelfa et de 0,15 à 0,23 à Béchar, confirmant le caractère sensitif de l'espèce plus marqué dans la région de Djelfa. Les coefficients d'autocorrélation plus élevés à Béchar $(0,45$ à 0,49$)$ confirment les valeurs obtenues sur les données brutes. Les 
valeurs d'EPS sont comprises entre 0,75 et 0,90 à Djelfa et 0,49 et 0,80 à Béchar. Les valeurs proches du seuil de 0,85 (Wigley et al., 1984) suggèrent que les arbres réagissent de façon homogène au sein des populations de Djelfa et de façon plus hétérogène au sein de celles de Béchar.

La réponse moyenne au climat a été évaluée par le calcul des coefficients de corrélation Bootstrap (BCC) entre les chronologies moyennes et les séries climatiques. L'objectif de cette approche est de déterminer les paramètres climatiques mensuels qui sont positivement ou négativement corrélés à l'épaisseur du cerne annuel. La fonction de réponse est considérée comme l'expression moyenne des facteurs limitants exercés sur les arbres d'un peuplement (Serre, 1973 ; Tessier, 1984).

Les résultats obtenus donnent une ébauche pour comprendre le comportement du pistachier vis-à-vis des conditions climatiques. Néanmoins, l'interprétation de ces résultats semble difficile, car nous ne disposons pas de données sur la physiologie et le fonctionnement de l'espèce. En effet, si l'interprétation peut, pour les stations de Djelfa, légitimement reposer sur ce que l'on connaît du calendrier de formation du cerne pour les espèces rencontrées dans le nord de l'Afrique du Nord (bioclimats humide à semiaride), en revanche, la transposition directe au climat saharien doit être menée avec beaucoup plus de prudence. Les résultats présentés dans les figures 5 et 6 montrent que la réponse du pistachier de l'Atlas varie davantage entre les régions qu'entre les stations d'une même région. À Djelfa se dégage une relation positive très significative avec les températures automnales (T11) pour Messaad et avec les températures hivernales et estivales (T1 et T8) pour Aïn Oussara. Les deux stations sont négativement corrélées avec les précipitations mensuelles d'octobre (P10) et positivement avec celles d'avril (P4). À Béchar, des différences significatives se dégagent entre les $B B C$ des deux stations. Les températures estivales (T7 et T8) sont positivement corrélées avec la croissance du pistachier à Djedida. À Oum Chegag, l'épaisseur des cernes est positivement corrélée aux températures hivernales (T1) et négativement à celles du printemps (T4). Une relation positive se distingue avec les précipitations automnales ( $\mathrm{P} 10$ ), printanières ( $\mathrm{P} 3$ et P4) et estivales (P7) à Djedida. Enfin, à Oum Chegag, la croissance radiale du pistachier de l'Atlas est positivement corrélée aux précipitations de septembre (P9) à août (P8) à l'exception du mois de mai (P5).

Les précipitations enregistrées en octobre de l'année précédente (P10) jouent un rôle négatif sur l'épaisseur des cernes dans les stations de Djelfa et positif pour les stations de Béchar. À Béchar, les pluies d'automne supérieures à la moyenne peuvent contribuer de façon notable à reconstituer le stock hydrique dans le sol disponible pour la phase ultérieure de croissance par une bonne mise en condition physiologique des plantes (Becker, 1977). Notons le rôle positif des précipitations de l'année en cours qui commencent dès février à Oum Chegag, mars à Djedida et avril pour les stations de Djelfa. Cette relation laisse penser que si la croissance radiale démarre probablement en avril à Djelfa, en revanche, la croissance démarre peut-être plus tôt à Béchar pour s'interrompre vers la fin avril et reprendre à la faveur d'orages très localisés, dans le courant du mois d'août. Ainsi, la croissance radiale du pistachier est positivement influencée par les précipitations de l'année en cours, enregistrées en juillet à Djedida et août à Oum Chegag. Ceci confirme que, contrairement à Djelfa où aucune activité cambiale de l'espèce n'est enregistrée, le pistachier ne possède pas une période de dormance estivale à Béchar. L'arbre profite des pluies orageuses de l'été et continue sa période de croissance. Mooney et Kummerow (1981) notent que, dans la région à climat de type méditerranéen de la Californie, la croissance des plantes est liée à la profondeur du système racinaire et au moment où l'eau devient disponible. Le système racinaire du pistachier de l'Atlas est très vigoureux, pouvant atteindre $6 \mathrm{~m}$ de profondeur. Cette vigueur lui permet d'occuper les fentes de rochers et les falaises, les terrains plats sur sols profonds ou très rocailleux, les lits d'oueds ou les grandes dépressions temporairement humides (Quézel et Médail, 2003). Au niveau des lits d'oueds, nous avons constaté que la longueur des racines latérales du pistachier peut atteindre plus de $20 \mathrm{~m}$, ce qui permet à l'arbre de profiter de l'eau mise à sa disposition sous des formes diverses (pluie, brouillard, rosée).

La relation négative avec les températures d'avril à Oum Chegag trouverait une explication dans le fait que des températures élevées seraient défavorables au bilan hydrique des pistachiers et qu'inversement, des températures relativement basses réduiraient l'évapotranspiration. Les corrélations positives obtenues au mois d'août (Aïn Oussara à Djelfa) et en juillet et août (Djedida à Béchar) ne peuvent être liées qu'à l'activité du cambium stimulée par les pluies de cette période. Belhadj (2007) souligne le rôle prédominant de la pluviométrie et du quotient d'aridité dans l'évolution de la variabilité des caractères morphologiques du pistachier de l'Atlas dans les différents habitats. Des adaptations xérophytiques ont été relevées au niveau de la morphologie de la surface épidermique et des stomates de cette espèce dans les stations les plus sèches. En outre, de nombreux auteurs affirment que la végétation méditerranéenne peut poursuivre la transpiration et la photosynthèse sous le stress lié à la sécheresse, et interrompre ces processus par la fermeture des stomates dans des conditions extrêmes de sécheresse (Zhang et Romane, 1991). L'épaisseur importante de son écorce représente une autre stratégie adaptative qui peut expliquer les corrélations positives entre la croissance radiale du pistachier de l'Atlas et les températures estivales. Cette alternative a été signalée par Cherubini et al. (2003) pour Arbutus unedo dans le Lajatico (Toscane, Italie).

Les résultats obtenus pour les coefficients de corrélation confirment le signal climatique présent dans l'expression des années caractéristiques au cours desquelles les températures supérieures à la moyenne agissent positivement sur la croissance radiale de l'espèce, surtout dans les deux stations de Béchar. Selon Baas (1976), les adaptations physiologiques peuvent modifier la réponse du cambium au climat au niveau de l'arbre et de l'espèce, de sorte que des différences de réponses de l'activité du cambium peuvent exister entre les espèces et entre les individus de la même espèce. 


\section{Conclusion}

L'étude de la croissance radiale et de la sensibilité au climat du pistachier de l'Atlas (Pistacia atlantica Desf.) par l'approche dendroécologique montre que la réponse de l'espèce à la variabilité climatique des deux régions est concrétisée par les valeurs élevées du coefficient de sensibilité moyenne. La distinction des années caractéristiques a permis de mettre en évidence le rôle des précipitations dans la croissance radiale du pistachier. Les années les plus sèches sont généralement les plus défavorables pour la croissance. En revanche, les années humides correspondent à des fortes croissances. L'étude de la variabilité interannuelle de la croissance, mesurée par le coefficient de sensibilité moyenne, s'est avéré très informative. L'analyse de la sensibilité moyenne obtenue pour les quatre séries chronologiques montre que le pistachier de l'Atlas peut être considéré comme sensible aux variations climatiques et représenter une mémoire biologique fiable pour les études dendroécologiques. Les fonctions de réponse montrent la prépondérance des précipitations dans le déterminisme de l'accroissement radial du pistachier de l'Atlas. Par contre, les températures n'exercent qu'un effet complémentaire. Dans les relations cerne-climat, le biotope joue un rôle majeur. Les résultats obtenus confirment que le pistachier de l'Atlas manifeste des traits d'adaptation en réponse au climat en modulant sa croissance en fonction des précipitations d'une extrême variabilité. Cette adaptation traduit sa très grande résistance aux changements globaux, notamment climatiques. En effet, les changements climatiques peuvent dépasser la capacité des espèces à s'adapter, particulièrement dans les zones vulnérables et menacées par la désertification telles les régions arides et semi-arides. De nouvelles données indiquent que beaucoup d'espèces dotées de capacités d'adaptation limitées encourraient des risques très élevés si la température moyenne globale augmentait de $+2{ }^{\circ} \mathrm{C}$. Dans certaines régions, des forêts entières pourraient disparaître de leur aire de répartition et être remplacées par une nouvelle diversité d'essences. Certaines espèces s'adapteront aux nouveaux climats, et d'autres sont déjà adaptées. Parmi ces espèces, le pistachier de l'Atlas, qui est un arbre rustique et autochtone des régions arides et semi-arides et se régénère, se développe dans les endroits les plus arides où peu d'espèces d'arbres peuvent s'établir et se développer. La connaissance de sa sensibilité et de ses seuils de réponse au climat est indispensable pour appréhender son comportement futur et les conséquences sur sa répartition spatiale. Ainsi, les mesures susceptibles de favoriser l'adaptation des écosystèmes résident dans l'amélioration de la gestion de l'occupation des sols, et la plantation d'espèces résistantes et adaptées. Cependant, l'étude effectuée ne concerne qu'une région bien limitée de l'Algérie. Notre perspective est d'élargir l'échantillonnage à d'autres régions et d'augmenter le nombre de populations échantillonnées sur toute l'aire géographique de cette espèce afin de fournir une vision à grande et moyenne échelle de son comportement.

\section{Remerciements}

Nous remercions Mohamed Said Guettouche, directeur du Laboratoire de géographie et aménagement du territoire (USTHB), pour son aide dans l'application du programme R.

\section{Références bibliographiques}

Amara M., 2014. Contribution à l'étude des groupements à Pistacia atlantica subsp. atlantica dans le Nord-Ouest algérien. Thèse de doctorat, Université Abou Bekr Belkaid Tlemcen, Algérie, $214 \mathrm{p}$.

Baas P., 1976. Some functional and adaptive aspects of vessels member morphology. In: Baas P., Bolton A. J., Catling D. M. (eds). Wood structure in biological and technological research. La Haye, Pays-Bas, Leiden University, Leiden Botanical Series, 3: 157-181.

Bagnouls F., Gaussen H., 1953. Saison sèche et indice xérothermique. Bulletin de la Société d'Histoire Naturelle, Toulouse, 88 : 193-239.

Becker M., 1977. Contribution à l'étude de la transpiration et de l'adaptation à la sécheresse des jeunes plants résineux. Exemple de 3 sapins du pourtour méditerranéen (Abies alba, A. nordmanniana, A. numidica). Annales des Sciences Forestières, 34 (2) : 137-158.

Becker M., 1989. The role of climate on present and past vitality of silver fir forests in the Vosges mountains of northeastern France. Canadian Journal of Forest Research, 19: 1110-1117.

Belhadj S., 2007. Étude éco-botanique de Pistacia atlantica Desf. (Anacardiaceae) en Algérie, préalable à la conservation des ressources génétiques de l'espèce et sa valorisation. Thèse de doctorat d'État en sciences agronomiques, Université Mouloud Mammeri Tizi Ouzou, Algérie, 183 p.

Boudouaya M., Benhassaini H., Bendimered-Mouri F. Z., Mothe F., Fournier M., 2015. Évaluation de la durabilité naturelle du bois de Pistacia atlantica Desf. du Nord de l'Algérie. Bois et Forêts des Tropiques, 325 (3) : 49-58. http://bft. cirad.fr/cd/BFT 325 49-58.pdf

Bréda N., Granier A., Aussenac G., 2004. La sécheresse de 2003 dans le contexte climatique des 54 dernières années : analyse écophysiologique et influence sur les arbres forestiers. Revue Forestière Française, 56 (2) : 109-131.

Burnel L., Pélissier C., 2009. Méthode de préparation d'échantillons de bois feuillus pour utilisation en dendrochronologie. Cahiers Techniques de l'Inra, $66:$ 5-12. 
Cherubini P., Gartner B. L., Tognetti R., Bräker O. U., Schoch W., Innes J. L., 2003. Identification, measurement and interpretation of tree rings in woody species from mediterranean climates. Biological Reviews, 78: 119-148.

Cook E. R., 1985. A time series analysis approach to tree ring standardization. Tucson, AZ, USA, University of Arizona, Faculty of the School of Renewable Natural Resources, 183 p.

Cook E. R., Kairiukstis L. A. E., 1990. Methods of dendrochronology: Applications in the environmental sciences. Dordrecht, Pays-Bas, Kluwer Academic Publishers, 408 p.

Emberger L., 1938. Les arbres du Maroc et comment les reconnaître. Paris, France, Édition Larousse, 317 p.

Fritts H. C., 1976. Tree rings and climate. Londres, Royaume-Uni, Academic Press, 567 p.

Guiot J., 1991. Methods and programs of statistics for paleoclimatology and paleoecology. Quantification des changements climatiques : Méthodes et programmes. Monographie 1. INSU, PNEDC, $253 \mathrm{p}$.

Hassini N., Abderrahmani B., Dobbi A., 2015. Changement climatique et phénomènes extrêmes : action symbiotique du climat et du milieu naturel dans l'occurrence de la sécheresse en Algérie. In : XXVIII Colloque de l'Association internationale de climatologie, Liège, Belgique, 503-508.

IPCC, 2014. Groupe d'experts intergouvernemental sur l'évolution du climat. Chapitre 22 : Afrique. Résumé. OMM, PNUE, $4 \mathrm{p}$.

Mérian P., 2012a. POINTER et DENDRO : deux applications sous $R$ pour l'analyse de la réponse des arbres au climat par approche dendroécologique. Outils et méthodes. Revue Forestière Française, 64 (6) : 789-798.

Mérian P., 2012b. Variations spatio-temporelles de la réponse au climat des essences forestières tempérées: quantification du phénomène par approche dendroécologique et influence de la stratégie d'échantillonnage Thèse de doctorat, Institut des sciences et industries du vivant et de l'environnement, (AgroParisTech), France, 451 p.

Monjauze A., 1980. Connaissance du bétoum Pistacia atlantica Desf. Biologie et forêt. Revue Forestière Française, 4 : 357-363.

Mooney H. A., Kummerow J., 1981. Phenological development of plants in mediterranean-climate regions. In: di Castri F., Goodall D. W., Specht R. L. (eds). Mediterranean-type Shrublands. Amsterdam, Pays-Bas, Elsevier Scientific Publishing Company, Ecosystems of the World 11, 303-307.

Pouget M., 1977. Cartographie des zones arides : géomorphologie, pédologie, groupements végétaux, aptitude du milieu à la mise en valeur à $1 / 100000$ : région de Messaad-Ain El Ibel (Algérie). Notice explicative $n^{\circ}$ 67. Paris, France, ORSTOM, $90 \mathrm{p}$.

Quézel P., Médail F., 2003. Écologie et biogéographie des forêts du bassin méditerranéen. Paris, France, Elsevier, coll. Environnement, $571 \mathrm{p}$.
R Development Core Team, 2011. R: A language and environment for statistical computing. Vienne, Autriche, R Foundation for Statistical Computing.

Safar W., 1994. Contribution à l'étude dendroécologique du pin d'Alep (Pinus halepensis Mill.) dans une région semiaride d'Algérie : l'Atlas Saharien (Ouled Naïl, Aurès, Hodna). Thèse de doctorat, Université d'Aix-Marseille III, France, $215 \mathrm{p}$.

Schweingruber F. H., 1996. Tree rings and environment: Dendroecology. Berne, Suisse, Paul Haupt, 609 p.

Schweingruber F. H., Eckstein D., Serre-Bachet F., Brakero U., 1990. Identification, presentation and interpretation of event years and pointer years in dendrochronology. Dendrochronologia, 8: 9-38.

Serre F., 1973. Contribution à l'étude dendroclimatologique du pin d'Alep (Pinus halepensis Mill.). Thèse de docteur ès sciences naturelles. Université d'Aix-Marseille III, France, $244 \mathrm{p}$.

Stokes M. A., Smiley T. L., 1968. An introduction to tree ring dating. Chicago, IL, USA, The University of Chicago Press, $73 \mathrm{p}$.

Tabet Aoul M., 2008. Impacts du changement climatique sur les agricultures et les ressources hydriques au Maghreb. Note d'alerte du CIHEAM, 48.

Tessier L., 1984. Dendroclimatologie et écologie de Pinus silvestris L. et Quercus pubescens Willd. dans le sud-est de la France. Thèse de doctorat, Université d'Aix-Marseille III, France, $275 \mathrm{p}$.

Wigley T. M., Briffa K. R., Jones P. D., 1984. On the average value of correlated time series, with applications in dendroclimatology and hydrometeorology. Journal of Climate and Applied Meteorology, 23: 201-213.

Zhang S. H., Romane F., 1991. Variations de la croissance radiale de Quercus ilex L. en fonction du climat. Annales des Sciences Forestières, $48:$ 225-234.

Zohary M., 1996. The genus Pistacia L. In: Padulosi S., Caruso T., Barone E. (eds). Taxonomy, distribution, conservation and uses of Pistacia genetic resources. Proceedings of the IPGRI Workshop, 29-30 June 1995, Palermo, Italy, 1-11. 